\title{
Study on the Characteristics of Electro Hydraulic Force Servo System with Friction and its Compensation
}

\author{
Li Jianying ${ }^{1, ~ a, ~ Z h a o ~ Z h o n g q i a n g ~}{ }^{1, b}$, Wang Yanwei ${ }^{2, ~ c}$, Li Shiming ${ }^{1, d}$, Dong \\ Fatang $^{1,}$ and Gao Bingwei $i^{1, f}$
}
${ }^{1}$ School of Mechanical and Power Engineering, Harbin University of Science and Technology, Harbin,Heilongjiang, China 150080

\author{
${ }^{2}$ School of Finance, Harbin University of Commerce, Harbin, Heilongjiang,China 150028 \\ aljyhit@163.com(J. Y. Li), b425297774@qq.com, cwywnancy@163.com,d314266672@qq.com, \\ e1805382297@qq.com, f545667967@qq.com.
}

\begin{abstract}
Keywords: Electro hydraulic force servo system, Friction force, Compensation, Nonlinear factors, Parameter variation, The system self compensation controller, Experimental research

Abstract. The purpose of this paper is to analyze the characteristics of electro hydraulic force servo system, at the same time, considering the serious disturbance of the friction force in the electro hydraulic force servo system, the nonlinear factors in the controlled object are analyzed, then we consider that the fact is the problem that the range of parameter variation is large and the model is difficult to be precise.

In this study, the classical control theory and linear dynamics theory are used to analyze the system; meanwhile, the nonlinear dynamic theory is adopted to solve the structure and parameters of nonlinear dynamics problems of electric hydraulic force servo system.

In this study, we focus on the influence law of nonlinear friction on the dynamic characteristics of electro hydraulic force servo system, and reveal deeply the typical nonlinear dynamic behavior of the system. Finally, we design the system self compensation controller, and it can effectively compensate the considering problem of system friction disturbance.

After observing the external friction force of the system, we use the system self compensation controller, to carry out the dynamic compensation for it. At the same time, through the theoretical analysis and experimental study, the suppression of the friction force disturbance by the system self compensation controller in the electro hydraulic force servo system is studied.
\end{abstract}

\section{Introduction}

Electro hydraulic servo system has the advantages of quick response, easy adjustment of parameters, large power to volume ratio, long self lubrication life and so on, It has been widely used in heavy vehicles, ships, aircraft, engineering machinery, weapon systems and so on[1]. The research of electro-hydraulic servo system has been carried out from two aspects: one of them is that the basic components research of electro hydraulic servo system, including servo valve and hydraulic cylinder, the other of them is research on control method and application technology of electro hydraulic servo system; they all have made corresponding research results[2]. The system integration technology based on electro hydraulic servo system has become a hot topic in this field, The technology let electro hydraulic servo system technology and computer application technology, linear control theory, electronic information technology, sensor technology, measurement mechanism design and analysis of modern control theory and other to discipline together, and it has been a great development $[3,4]$. Hydraulic cylinder of basic element research mainly aimed at the hydraulic cylinder low friction force, lateral force resistant, etc[5]. There is generally friction force between the piston and cylinder, during low speed, it shows strongly nonlinear effect, it can lead to crawl, vibration and unstable phenomenon to appear, and it will seriously affect the control effect of the system $[6,7,8]$. Due to fluid flow characteristics of electro-hydraulic servo system, compressibility, viscosity and high cleanliness requirements, and it is susceptible to the influence of the environment, such as temperature, pressure, etc, so electro hydraulic servo system dynamic performance in the process of movement are relatively 
complex, appear easily crawl vibration and noise, impact and work such as irregular situation, and it's easy to have a system can't work stable, poor dynamic characteristics.

This paper adopts the classical control theory and linear dynamics theory analysis system and the nonlinear dynamics theory, aiming at the electro hydraulic force servo system nonlinear dynamic problems of electro hydraulic servo system, the influence law of nonlinear friction force on dynamic characteristics of electro hydraulic force servo system is studied. It reveals deeply the typical nonlinear dynamic behavior of the system, and the purpose of this paper is to reveal the mechanism and inducement of nonlinear vibration and creep of electro hydraulic force servo system, in this way, the dynamic characteristics of the integrated analysis system becomes closer to reality. The above work is studied and verified by theoretical analysis and experimental research.

\section{Mathematical equation and dynamic model of electro hydraulic force servo system}

The actuator of electro hydraulic force servo system is cylinder controlled by electro hydraulic servo valve, in this paper, double acting with double output rod servo hydraulic cylinder is adopted. Its working principle is shown as Figure 1, The dynamics equation of it is shown as [9]:

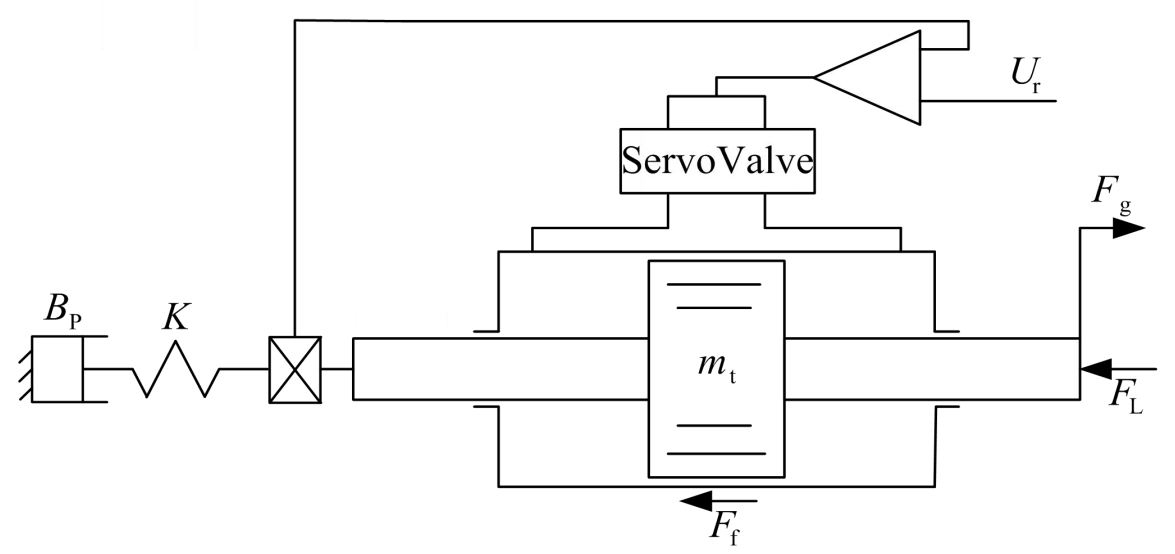

Figure 1 Schematic diagram of electro hydraulic force servo system

$$
A_{P} P_{L}=m_{t} \ddot{X}_{P}+B_{P} \dot{X}_{P}+K X_{P}+F_{f}+F_{L} \text {. }
$$

Where: $m_{t}$-Equivalent mass of piston and inertia load ; $X_{P}$-Piston displacement; $B_{P}$-Viscous damping coefficient of piston and load; $K$-Elastic coefficient of load; $F_{f}$-Friction force; $F_{L}$-Any external load force acting on the piston; $P_{L}$-Hydraulic cylinder two chamber load pressure; $A_{P}$-Effective area of hydraulic cylinder piston.

It can be seen from the literature that the relationship between friction force and velocity is Stribeck curve, it is the result of the study of the relationship between the velocity, the normal load and the viscosity of the lubricant and the friction coefficient by the German scholar Stribeck. The curve describes four areas, it is respectively a zone (1) static friction zone, a region (2) boundary lubrication region, a region (3) mixed lubricationregion and a region (4) hydrodynamic lubrication region, the mathematical model can be described by formula (2) and formula (3)[10]. The viscous friction force is proportional to the velocity and is a linear function of the velocity. When the system is operating at a low speed, the friction force decreases exponentially with the increasing velocity from the Static friction force to the Coulomb friction force [11]. The Stribeck model is nonlinear, which depends on the instantaneous speed value of the system.

$$
F_{f}=\left\{\begin{array}{rll}
F(v) & v \neq 0 & \\
F_{e} \operatorname{sgn}\left(F_{e}\right) & v=0 & \left|F_{e}\right|<F_{j} \\
F_{j} \operatorname{sgn}\left(F_{e}\right) & v=0 & \left|F_{e}\right| \geq F_{j}
\end{array}\right.
$$




$$
F_{v}=\operatorname{sgn}(v)\left[F_{k}+\left(F_{j}-F_{k}\right) \exp \left(-\mid \frac{v}{v_{s}}\right)^{\tau}\right]+B v .
$$

Where: $v$-Speed; $F_{e}$-External force; $F_{j}$-Maximum static friction force; $F_{k}$-Coulomb friction; $v_{s}$-Stribeck speed; $B$-Viscous friction coefficient; $\tau$-Empirical parameter, Its value is generally between 0.5-2.0.

When applied here, in order to facilitate marking, $v$ will be set directly to the piston rod movement speed, we suppose that $\dot{Y}$ is the change value of movement velocity of piston displacement $X_{P}$ nearby, then the friction force is expanded into Taylor Series in the vicinity of $X_{P}$ nearby:

$$
F_{f}(v+\dot{Y})=F_{f}(v)+\dot{F}_{f}(v) \dot{Y}+\frac{\ddot{F}_{f}(v)}{2 !} \dot{Y}^{2}+\frac{\dddot{F}_{f}(v)}{3 !} \dot{Y}^{3}+\Delta\left(\dot{Y}^{3}\right) .
$$

We respectively use $B_{1}, B_{2}$ and $B_{3}$ to replace $\dot{F}_{f}(v), \frac{\ddot{F}_{f}(v)}{2 !}$ and $\frac{\dddot{F}_{f}(v)}{3 !}$. As the piston is reciprocating motion, we can limit the scope of work to a certain operating point, higher order infinitesimal can be ignored, then the formula (4) friction force can approximate be expressed as:

$$
F_{f}(v+\dot{Y})=F_{f}(v)+B_{1} \dot{Y}+B_{2} \dot{Y}^{2}+B_{3} \dot{Y}^{3} .
$$

The effect of friction force is different from that of the working point on the Stribeck curve [12]: Firstly, when the working point is located in area (1), Stribeck curve slope is very positive, the effect of the damping moment increased sharply, So that the fast of the dynamic response of the system is contained; Secondly, when the working point is located in area (4), the friction force is linear, its effect is equivalent to increasing the damping of the system; Thirdly, when the operating point is located in the area (2) or area (3), the friction has nonlinear time varying characteristics, especially when the system is in a state of less damping or even negative damping, it will seriously affect the stability of the system, and it can lead to unstable operation of the system and may produce self-excited vibration with limit cycle. Therefore, the focus of the discussion is that when the working point is located in the region (2) or region (3), the friction force exhibits nonlinear time-varying characteristics.

In order to study the influence of nonlinear friction force on the dynamic characteristics of the system, when the operating point is located in the area (2) or area (3), $B_{1}$ is negative, $B_{2}$ is approaches zero, $B_{3}$ is not less than zero, then the characteristic of formula (1) can be expressed as:

$$
m_{t} \ddot{Y}+\left(B_{P}+B_{1}\right) \dot{Y}+K Y+B_{3} \dot{Y}^{3}=A_{P} P_{L}-\left(F_{f}(v)\right.
$$

As the oil source as the power source output pressure pulsation, pipeline pressure pulsation, the influence of valve orifice flow pressure nonlinearity, the pressure of the hydraulic oil flowing into the hydraulic cylinder has high frequency fluctuation[13], the law obeys the law of simple harmonic vibration, therefore, the input to the right of the equal sign in the equation (6) can be approximated as $F \sin \left(\omega t+\varphi_{0}\right)$, it is the excitation source of the system[14]. Where, $F$-Exciting force; $\omega_{t}$-Exciting angle frequency; $\varphi_{0}$-Initial phase angle of exciting force, the formula (6) can be changed to:

$$
m_{t} \ddot{Y}+\left(B_{P}+B_{1}\right) \dot{Y}+K Y+B_{3} \dot{Y}^{3}=A_{P} P_{L}-F \sin \left(\omega t+\varphi_{0}\right)
$$

For servo valve controlling hydraulic cylinder system, piston displacement $X_{P}$ mathematical expressions can be expressed as:

$$
m_{t} s^{2} X_{P}+\left(B_{P}+B_{1}\right) s X_{P}+K X_{P}+B_{3} s^{3} X_{P}=A_{P} P_{L}-F \sin \left(\omega t+\varphi_{0}\right)
$$

The following is to establish the valve flow equation, the selected slide valve is zero open four side slide valve, the four throttling windows are matched and symmetrical, the oil supply pressure and oil return pressure are constant and the oil return pressure is zero, the flow equation can be expressed asa[15]:

$$
Q_{L}=K_{q} x_{v}-K_{c} P_{L}
$$


Where: $K_{q}$-Flow gain, $K_{c}$-Flow pressure coefficient, $x_{v}$-Spool displacement, $P_{L}$-Load pressure.

Secondly, the flow continuity equation of hydraulic cylinder is established, under conditions of satisfaction, namely, the connection of the valve and the hydraulic cylinder is symmetrical and short and thick, the pressure in the working chamber is equal to each other, oil temperature and bulk modulus of elasticity is constant, the internal and external leakage of the hydraulic cylinder is laminar flow, the flow continuity equation of hydraulic cylinder can be expressed as [16]:

$$
Q_{L}=A_{P} s X_{P}+C_{t p} P_{L}+\frac{V_{t}}{4 \beta_{e}} s P_{L}
$$

Where: $C_{t p}$-Total leakage coefficient of internal and external leakage of hydraulic cylinder, $V_{t}$-Total compression volume of hydraulic cylinder, $\beta_{e}$-Effective bulk modulus.

The block diagram of the power mechanism is shown in Figure 2:

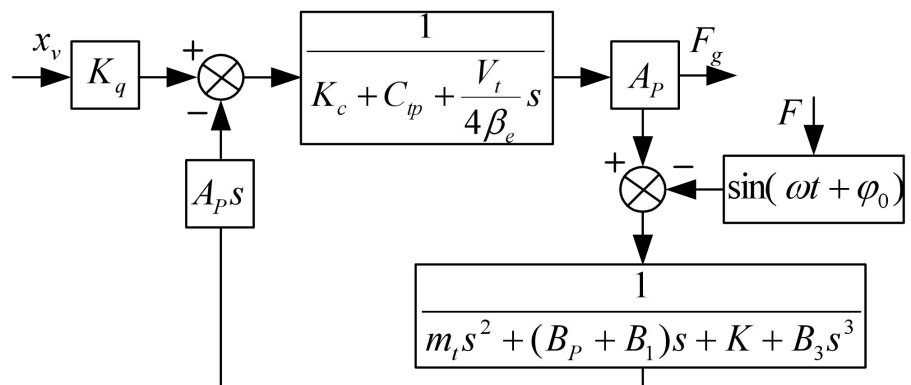

Figure 2 The block diagram of the power mechanism

Comparison components bias voltage signal is[17]:

$$
U_{e}=U_{r}-U_{p}
$$

Where: $U_{r}$ and $U_{p}$ are the instruction voltage signal and the feedback voltage signal respectively.

Due to the high response frequency of the displacement sensor, its transfer function equation can be expressed as:

$$
U_{p}=K_{p} X_{P}
$$

Where: $K_{p}$-Displacement sensor gain.

As the servo amplifier is an electrical component, its response frequency is high, so its dynamic performance can be ignored, and its output current can be expressed as:

$$
\Delta I=K_{a} U_{e}
$$

Where: $K_{a}$-Servo amplifier gain.

In order to simplify the system model and can focus on the characteristics of electro hydraulic force servo system under the action of friction force, so the electro hydraulic servo valve of the system can be regarded as proportional link, namely:

$$
G_{s v}(s)=\mathrm{X}_{\mathrm{v}} / I=K_{s v}
$$

Where: $K_{s v}$-Electro hydraulic servo valve flow gain.

\section{System self compensating controller}

Through the analysis of the transfer function and block diagram model of the system, and considering the interference of the friction force in the electro hydraulic servo system, and considering the nonlinear factors and the big range of parameter variation in the controlled object, In this study, the system self compensation controller is designed and adopted, and it is used to effectively deal with the above conditions. 
The idea of observer in modern control theory is adopted in the system self compensation controller. This method does not require high accuracy of the mathematical model of the controlled object, by observing the state variables of the system by the extended state observer, it can also observe the external interference friction force of the system, then the targeted dynamic compensation is carried out, therefore, the controller has strong ability to resist external interference, and it can effectively restrain the influence of friction force on the stability of the system and other comprehensive characteristics[18].

By the above analysis, the electro hydraulic force servo system studied is four order system, so the structure of the system self compensation controller is shown in Figure 3. The tracking differentiator extended state observer and feedback control rate of the system self compensation controller can be described by the following equation [19]:

TD-Tracking differentiator, FCL-Feedback control law, ESO-Extended state observer

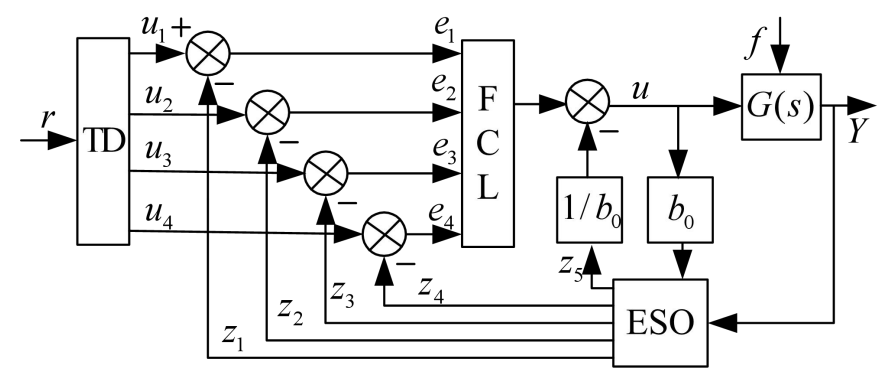

Figure 3 The structure of the system self compensation controller

Tracking differentiator in system self compensating controller, is mainly used to solve the contradiction between the response speed and the overshoot of the system, and avoid excessive overshoot of the system, its specific algorithm is:

$$
\left\{\begin{array}{c}
l(k)=\operatorname{fhan}\left(u_{1}(k)-r(k), u_{2}(k), n, h_{0}\right) \\
u_{1}(k+1)=u_{1}(k)+h u_{2}(k) \\
u_{2}(k+1)=u_{2}(k)+h u_{3}(k) \\
u_{3}(k+1)=u_{3}(k)+h l(k) \\
u_{4}(k+1)=l(k)
\end{array}\right.
$$

Where: fhan $\left(u_{1}(k)-r(k), u_{2}(k), n, h_{0}\right)$ is fast optimal control function, $n$ is the velocity factor, and it determines the speed of tracking a given signal, $h$ is an integral step, $h_{0}$ is filter factor.

Secondly, the extended state observer algorithm is:

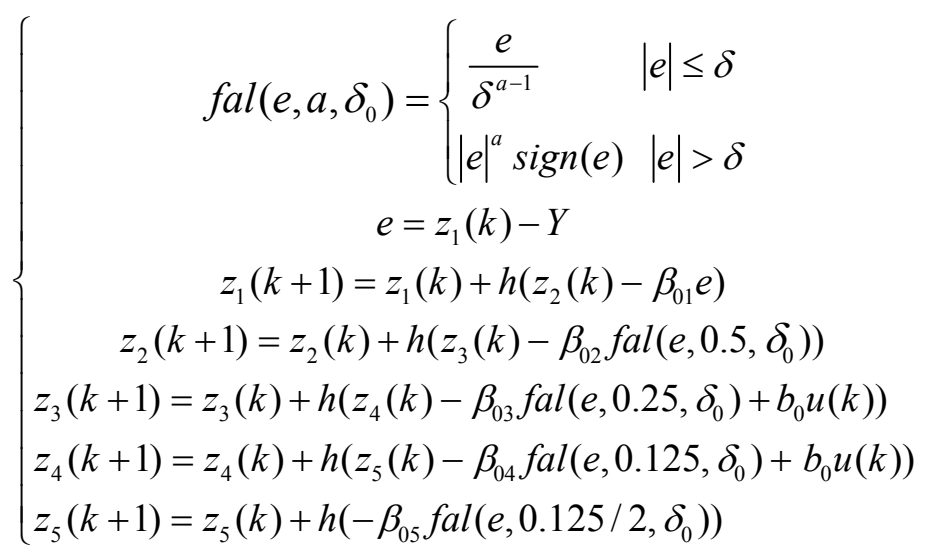

Where: $\mathrm{fal}\left(e, a, \delta_{0}\right)$ is nonlinear function; $\beta_{01}, \beta_{02}, \beta_{03}, \beta_{04}, \beta_{05}, \delta_{0}, b_{0}, h$ are all planning tuning parameter. 
Feedback control law generates the control signals of electro hydraulic force servo system $u$, and it compensates for the interference signal in real time, and it can realize feedback linearization of dynamic system. The algorithm of feedback control law is

$$
\left\{\begin{array}{c}
e_{1}=u_{1}(k)-z_{1}(k) \\
e_{2}=u_{2}(k)-z_{2}(k) \\
e_{3}=u_{3}(k)-z_{3}(k) \\
e_{4}=u_{4}(k)-z_{4}(k) \\
u_{0}=\beta_{11} f a l\left(e_{1}, a_{1}, \delta_{1}\right)+\beta_{12} f a l\left(e_{2}, a_{2}, \delta_{1}\right)+\beta_{13} f a l\left(e_{3}, a_{3}, \delta_{1}\right)+\beta_{14} f a l\left(e_{4}, a_{4}, \delta_{1}\right) \\
u(k)=u_{0}-z_{5}(k) / b_{0}
\end{array}\right.
$$

Where: $\beta_{11}, \beta_{12}, \beta_{13}, \beta_{14}, a_{1}, a_{2}, a_{3}, a_{4}, \delta_{1}, b_{0}$ are all planning tuning parameter.

Through a large number of simulation studies, and based on debugging experience, and at the same time we get the best satisfied control effect, the controller parameters are identified.

\section{Experimental study of electro hydraulic force servo system and system self compensation control}

The experimental table carrying experiment study is shown in Figure 4. The whole experiment platform includes computer, and the computer is used for creating the real time signal, adjusting the signal source, and the online and offline processing of the collected data; the whole experiment platform includes electric control panel too, it is used for analog signal processing, power amplification, signal retention, manual tuning, etc.; the whole experiment platform includes the main experimental platform as well(including experimental bench, it is used to support hydraulic cylinder, guide rail, load, etc.; actuator valve control cylinder; the force sensor is used to measure the output force of the piston rod; sliding guide rail for supporting mass tray; and inertial load, flexible link, etc.) and Hydraulic oil source etc. other auxiliary equipment four parts.

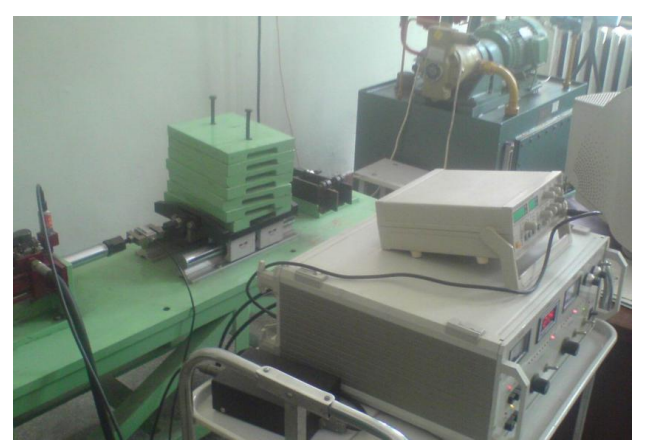

Figure 4 The experimental table of electro hydraulic force servo system

The operation and control software of the experimental platform is realized by using RCP (Rapid Control Prototype) technology [20], it is very convenient to construct the structure of real-time online controller and adjust the parameters of the controller, and can be quickly implemented on a physical entity. In the process of software running, changing the parameters of the model through online real time (whether alone or in combination), can verify or compare the effect before and after changing parameters, to optimize the parameters or determine the optimal range of parameters. It also has a powerful function is the ability to automatically generate executable code, thus, the researchers can quickly generate the machine code language which can be recognized and executed by the controller which is modularized or graphic, improve the human-computer interaction, and then the control strategy of block diagram can be transformed into the code that can be executed quickly and effectively, parameter adjustment and signal acquisition and analysis are very convenient [21]. In this paper, the realization of RCP is realized by the function provided by Matlab software platform. Under the support of the rapid control prototype system, the software development model of the control 
system is changed from the linear sequence model to the fast cyclic iteration model, which is easy to implement and has high reliability.

In order to verify the effectiveness of the system self compensation controller in the practical application of the electro hydraulic force servo system, designed in this paper, and the actual control effect of the self compensator adopted or not is compared. Experimental study was carried out about the effectiveness of the system self compensating controller on the electro hydraulic force servo system experiment platform in Figure 4. Figure 5 shows the $5 \mathrm{~Hz}$ sine response curve of the system under the action of the self compensating controller, the curves 1 and the curve 2 in Figure 5 are the input curve and the response output curve of the system. Figure 5 (a) is the experimental curve of the system without using the self compensation controller, Figure 5 (b) is the experimental curve of the system with the self compensating controller.

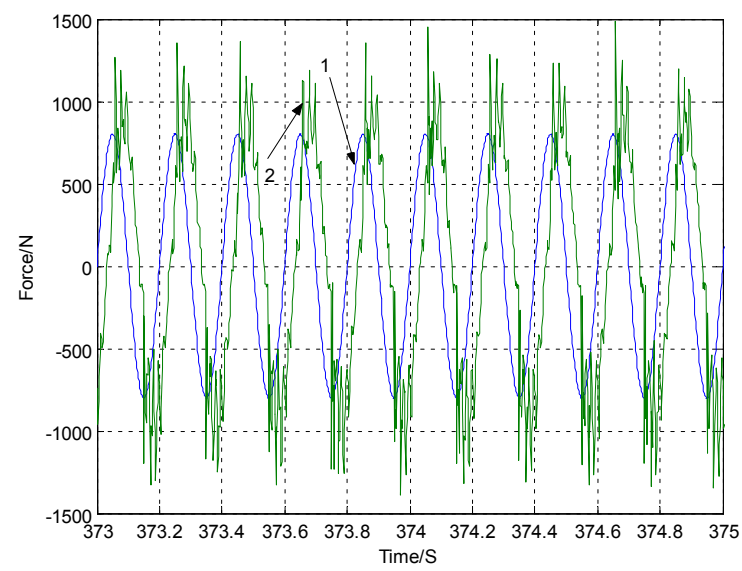

(a)

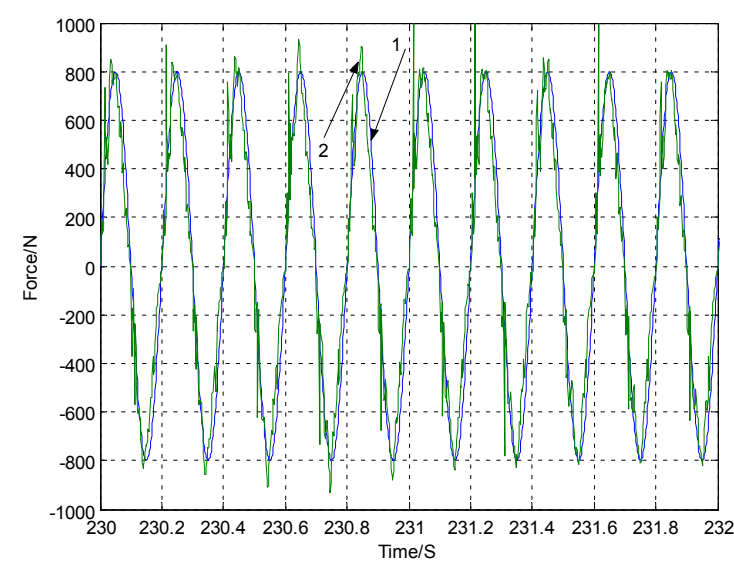

(b)

Figure 5 Experimental curve of electro hydraulic force servo system

Comparing Figure 5 (a) and (b), we can know when the piston rod moves to the limit position of both ends and the reversing process, the friction force in Figure 5 (a) is very large, the output value generally exceeds the input value of about $60 \%$, the output value of individual positions even more than 1 times the input value, and in the limit position there is obvious jitter crawling behavior. The friction force in the above position of Figure 5 (b) is also existence, but it has been weakened, the output value is generally close to the input value, crawling and jitter are also significantly weakened, so amplitude aspect the output signal can track the input signal well. On the other hand, in the tracking phase, the phase lag of a is obvious in Figure 5 (a), and it can be seen obviously that in the middle vicinity of the piston moving, the two sides have a severe delay jitter points, this shows that friction force is larger. But in Figure 5 (b) this phenomenon has basically disappeared, at this particular point, the curve is smooth, and the phase lag phenomenon is relieved. The two typical experimental phenomena show that there is serious friction force interference in electro hydraulic force servo system; consideration should be given to system modeling and system analysis, in order to avoid the large difference between the actual situation and theoretical analysis. At the same time, after using the system self compensating controller, the friction force interference of electro hydraulic force servo system is greatly suppressed, the crawling and chattering phenomenon is also obviously improved, and the dynamic performance, etc. comprehensive performance of the system is well guaranteed.

\section{Conclusions}

The friction force is universally exists in electro hydraulic force servo system, the friction force mainly exists between the piston and cylinder of the servo hydraulic cylinder and piston rod and cylinder head position, the main reason is to solve the sealing and guiding structure, the strong nonlinear effect of friction force is at low speed or in the direction change of high frequency motion, it can cause creeping, vibration and instability, and it seriously affects the control effect of the system. 
In this paper, the classical control theory and linear dynamics theory are used to analyze the system, at the same time, the nonlinear dynamic theory is adopted, aiming at the nonlinear dynamic problem of electro hydraulic force servo system, the mathematical equation and dynamics model of electro hydraulic force servo system are established. This paper also studies the design of the system self compensation controller, for the above characteristics, using the idea of observer in modern control theory, the external friction force of the system can be observed, then the dynamic compensation is carried out, which has strong ability to resist external interference. The theoretical analysis and experimental study show that the friction force in the electro hydraulic force servo system is greatly suppressed, crawling and shaking phenomenon also improved significantly, the dynamic performance of the system is well guaranteed, so the research above all in this paper can be used as reference for other related researches.

\section{Acknowledgements}

This work is supported by National Natural Science Foundation of China (51405113).

\section{References}

[1] Xing Zongyi, Zhang Yuan, Hou Yuanlong, Jia Limin(2009), "Modeling of Electrohydraulic System and Its Application”, Journal of System Simulation, Vol. 21 No. 6 : pp. 1719-1725.

[2] Han Junwei(2012), "Development and Application of Electro-hydraulic Servo-system", Machine Tool \& Hydraulics, Vol. 40 No. 2 : pp. 7-10.

[3] Yang Chifu, Han Junwei, Zheng Shutao(2012), "Dynamic Modeling and Computational Efficiency Analysis for a Spatial 6-DOF Parallel Motion System”, Nonlinear Dynamics, Vol. 67 No. 2 : pp. 1007-1022.

[4] Huang Qitao, Jiang Hongzhou, Han Junwei(2005), "Spacecraft Docking Simulation Using Hardware-in-the-loop Simulator with Parallel Platform", Journal of Chinese Mechanical Engineering, Vol. 18 No. 3 : pp. 415-418.

[5] Dasgupta K, Murrenhoff H(2011), "Modelling and Dynamics of a Servovalve Controlled Hydraulic Motor by Bondgraph", Mechanismand Machine Theory, Vol. 46 No.7 : pp. 1016-1035.

[6] Wang Linhong, Wu Bo, Du Runsheng(2007), "Nonlinear Dynamic Characteristics of Moving Hydraulic Cylinder”, Chinese Journal of Mechanical Engineering, Vol. 43 No. 12 : pp. 12-19.

[7] Yang Anyuan, Yang Xue(2004), "Research on Approaches to Weakening Vibration of Hydraulic System”, Chinese Hydraulics \& Pneumatics, No. 2 : pp. 51-53.

[8] Tang Rui, Zhang Qi(2011), "Dynamic Sliding Mode Control Scheme for Electro-hydraulic Position Servo System”, Procedia Engineering, No. 24 : pp. 28-32.

[9] Wang Chunxing(2011), "Hydraulic Control System”, Machinery Industry Press, Beijing.

[10]Zhang Congpeng, Liu Qiang(2008), "Friction Modeling and Compensation of Positioning Stage Driven by Linear Motors", Journal of Beijing University of Aeronautics and Astronautics, Vol. 34 No. 1 : pp. 47-50.

[11]Wen Ming, Wang Xinfang, Deng Zichen(2009), "Precise Response Arithmetic for Systems with Pseudo-viscous Frictional Energy Dissipator", Mechanical Science and Technology for Aerospace Engineering, Vol. 28 No. 12 : pp. 1584-1587.

[12]Xi Zhuyan, ZhangTao, Lu Changhou(2006), "Overview of Friction Compensation Control in Table Servo Feed Systems of CNC Tools", Modern Manufacturing Engineering, No. 1 : pp. 21-26. 
[13]Li Jianying, Shao Junpeng, Wang Zhongwen, Wu Bo, Han Guihua(2009), "Study of Electro-Hydraulic Force Servo Control System Based on Fuzzy Control”, Proceedings-2009 IEEE International Conference on Intelligent Computing and Intelligent Systems, : pp. 688-693.

[14]Chen Yushu, Tang Yun, Lu Qishao(2000), "Modern Analysis Methods from Nonlinear Dynamics", Science Press, : pp. 1-50.

[15]Li Hongren(1990), "Hydraulic Control System (Revised Version)”, National Defence Industry Press, : pp. 53-59.

[16]Shao Junpeng, Li Jianying, Wang Zhongwen, Han Guihua(2009), "Structure Compensation Control of Eliminating Super Fluous Force of Electro-hydraulic Load Simulator", Electric Machines and Control, Vol. 13 No.4 : pp. 586-591.

[17]Hua Qing, Jiao Zongxia, Wang Xiaodong, Wang Shaoping(2002), “Complex Mathematical Model of Electro-hydraulic Torque Load Simulator", Chinese Journal of Mechanical Engineering, Vol. 38, No.11, : pp. 31-35.

[18]Zheng Qing, Dong Lili, Lee Daehui(2009), “Active Disturbance Rejection Control for MEMS Gyroscopes", IEEE Transactions on Control Systems Technology, Vol. 17 No. 6 : pp. 1432-1438.

[19]Han Jingqing(2008), “Active Disturbance Rejection Control Technology: the Technology for Estimating and Compensating the Uncertainties”, National Defense Industry Press, : pp. 71-77.

[20]Ding Rongjun(2009), “The Development Status of Fast Control Prototype”, Electric Drive for Locomotives, No. 4, : pp. 1-4.

[21]Yang Xiangzhong, An Jinwen, Cui Wenge(2009), "The Application on Rapid Control Prototyping Simulation", Aerospace Control, Vol. 27 No. 2 : pp. 72-76. 